\title{
Aproximación al estado de la educación artística en las ciudades de Machala y Pasaje, Ecuador
}

\section{Approach to the state of arts education in the cities of Machala and Pasaje, Ecuador}

\author{
Enrique Espinoza Freire
}

Universidad Técnica de Machala, P.O. BOX 462 Machala - El ORO. Ecuador

DOI: https://doi.org/10.33017/RevECIPeru2015.0022/

\section{Resumen}

Las Conferencias mundiales y especialmente las latinoamericanas, en torno a la educación artística, ha sido un intento por incidir en la problemática de la enseñanza. Una de la iniciadora de ese ciclo de debates, proyectos y gestión programáticas en torno de esta educación, parecen estar enlazados a la realizada en Bogotá, del 28 al 30 de noviembre de 2005, bajo el slogan: "Hacia una Educación de Calidad: Retos y Oportunidades". La Conferencia convocó a 21 países, y el Comité Académico del evento envió el Documento Conceptual elaborado por la Oficina Regional de Cultura de la UNESCO y un Cuestionario para ser diligenciado conjuntamente por los delegados de educación y cultura. Aquel cuestionario ha sido un pretexto metodológico para realizar una mirada local a esta problemática, pues los pronunciamientos de la llamada "Declaración de Bogotá", siguen aún vigentes en nuestro país. Además de inspirar y constituir los fundamentos de las Metas Educativas 2021. Tal cuestionario facilita una reflexión acerca de los alcances, que necesariamente antecede a una introducción más integral, del programa de la educación artística, en el entramado institucional que corresponda. Lo significativo radica en que la identificación de oportunidades y retos, incluye la caracterización de los imaginarios instaurados que obstaculizan o potencian su instauración más o menos exitosa, por lo que, adaptándolo al contexto ecuatoriano, resulta una caracterización destinado a docentes en ejercicio, en las instituciones educativas de Educación Básica de la ciudad de Pasaje y Machala de Ecuador.

Descriptores: Educación Artística, Educación Básica, enseñanza del arte

\section{Abstract}

The world and especially Latin American, about arts education conferences has been an attempt to influence the problem of teaching. One of the initiator of this series of debates, projects and program management education around this appear to be linked to the one held in Bogota from 28 to 30 November 2005, under the slogan "Towards Quality Education: Challenges and Opportunities ". The Conference brought together 21 countries, and the Academic Committee of the event sent the concept paper prepared by the Regional Office for Culture of UNESCO and a questionnaire to be filled jointly by the delegates of education and culture. That questionnaire was a methodological pretext for a local look at this issue, because the pronouncements of the "Declaration of Bogota", are still in force in our country. Besides inspiring and constitute the foundations of the Educational Goals 2021. This survey provides a reflection on the scope, which necessarily precedes a more comprehensive introduction, the art education program in the appropriate institutional framework. What is significant is that the identification of opportunities and challenges, including the characterization of the imaginary in place that hinder or enhance its more or less successful establishment, so, adapting the Ecuadorian 
context, a characterization is aimed at practicing teachers in the Basic Education educational institutions of the city of Pasaje and Machala in Ecuador

\section{Keywords: Arts Education, Basic Education, teaching art}

\section{Introducción}

Continua siendo de interés el tema de la influencia del arte en la enseñanza formal de los infantes, pues a todos preocupa la eficacia con que se "aprende", en la llamada sociedad de la información, que acompañada por el arsenal tecnológico que le caracteriza ha contribuido a masificar las fuentes de información, cuestión que sin dudas ha hecho más dinámico el aprendizaje de los individuos.

Tal contexto ha reavivado los debates en torno a la problemática ya mencionada, no solo por los retos educacionales que en general enfrentan todas las sociedades de hoy, sino también, porque en consecuencia, uno de esos desafíos está relacionado al nivel de profundidad que exigen, los conocimientos que son impartidos a todos los estudiantes, lo cual ha obligado a ampliar los contenidos tradicionales de las asignaturas que apoyan una aprehensión más integral del universo, con nuevas relaciones socioculturales que los futuros hombres y mujeres habrán de enfrentar.

Desde entonces he observado como el aprovechamiento académico, sistema de evaluación al fin y al cabo, es concebido para medir más allá de lo bien realizado o aprendido por los alumnos. Han adquirido además importancia las asignaturas de mayor libertad creativa, fundamentalmente las de corte artístico-literario, al facilitar como ninguna otra el pronto discernimiento del mundo, por lo que no es casual que ahora su espectro de contenidos sea mayor [1]. Pues de lo que se trata, es que la escuela forme a quienes puedan repensar y cambiar el mundo, enseñándoles a diferenciar lo bueno de lo nocivo, a partir del desarrollo del pensamiento crítico; y la capacidad de elegir conscientemente valores y concepciones de vida, de acuerdo claro está, de las nociones de identidad nacional y cultural de los individuos.

Es lo que está detrás del interés que recibe la educación artística, y la consecuente pregunta, relacionada al reto particular que tiene este programa educativo como metodología pedagógica. ¿Tiene coherencia la forma en la que las artes son dispuestas en el estudio de educación básica, en el conjunto de materias y su propósito educativo, con la forma en la que se construye el mundo en la actualidad? [2]. También un centro tradicional de la iniciación escolar y por consiguiente sensible conductor de la educación básica.

Era de esperarse la preocupación internacional devenida en objetivo estratégico, desde el cual la UNESCO, promueve la inclusión de disciplinas artísticas, en la formación general del niño y del adolescente, al considerar que la educación artística: Contribuye al desarrollo de su personalidad, en lo emocional y en lo cognitivo; tiene una influencia positiva en su desarrollo general, en el académico y en el personal; Inspira el potencial creativo y fortalece la adquisición de conocimientos; estimula las capacidades de imaginación, expresión oral, la habilidad manual, la concentración, la memoria, el interés personal por los otros, etc.; contribuye al fortalecimiento de la autoconciencia y la identidad propia; concede a los niños y adolescentes canales de comunicación y autoexpresión; contribuye a la creación de audiencias de calidad favoreciendo el respeto intercultural[3].

Esta organización mundial ha reconocido la experiencia practica, resultante de la aplicación de la educación artística como instrumento de la enseñanza, solo que en el contexto sociocultural ya descrito, cada una de estas dimensiones cobran un especial significado. Por lo que no creo se discuta su importancia, ni a quien hay que enseñar el arte, sino que, en realidad a maestros y pedagogos les ha preocupado siempre más, el cómo hacerlo.

Problemática que tambien experimentó el modelo de la escuela tradicional, que se preocupó cómo enseñar el arte y el sistema de valores que les permitiera desarrollar aptitudes de apreciación artística, no tan sólo una materia escolar, sino una asignatura destinada a la formación del mundo espiritual de los niños, casi un rezo en los multiples manuales que intentaron una didáctica de la enseñanza de las artes, y aunque el paradigma nunca fue crear "artistas" masivamente, cierto rumbo tecnicista contaminó una educación artística, que terminó siendo una enseñanza formal del arte, más que la creatividad soñada, lo cual pareció una auténtica desconexión 
con una realidad que demandaba soluciones concretas, algo que hizo disminuir su importancia.

Según [4], tal cuestión puede corroborarse, en el hecho conocido, de que las primeras investigaciones publicadas sobre Educación Artística pegadas a los modelos tradicionales en las primeras décadas del siglo $\mathrm{XX}$, recurriendo sistemáticamente al uso de "test" y pruebas objetivas en estudios descriptivos, longitudinales y correlaciónales sobre capacidades artísticas (especialmente el talento para el dibujo y la capacidad creativa), y su relación con el rendimiento escolar general.

La evolución vendría alrededor de los 50 del siglo $X X$, la mayoría de los principales proyectos de investigación, se orientaron hacia el desarrollo de modelos curriculares innovadores, que abordaron la complejidad del aprendizaje artístico del sistema escolar, especialmente los años de escolaridad obligatoria [5].

Los últimos diez años del XX, significa el momento de consolidación de los principales temas y metodologías de investigación, y con ello el sistema de ideas que se impone en la actualidad [6], según éste autor los principales manuales de investigación publicados en los últimos años, abordan por lo general: primero, la Cultura Visual, que en el contexto de la Educación Artística, implica básicamente dos cosas, por una parte la ampliación del campo de fenómenos estudiados que no quedan circunscritos a las imágenes visuales reconocidas como artísticas, sino al conjunto de los acontecimientos visuales presentes en la vida cotidiana del alumnado, tales como los muñecos, carteles, pegatinas y tatuajes con los que los preadolescentes decoran su habitación, los parques de atracciones, o los envoltorios de los caramelos y chucherías; por otro, una reconsideración de los modos de interpretación de todas esas imágenes centrándose en los aspectos que son verdaderamente significativos en la configuración de la identidad personal y grupal del alumnado.

El segundo tema definitorio de las investigaciones en Educación Artística es el estudio en profundidad del dibujo y el arte infantil espontáneo de Hernández, 1995 [7], y Martínez, 2004[8]. Y el tercero El consenso de los organismos y asociaciones internacionales: Reforzar las identidades y valores personales y sociales y la diversidad cultural a través de la Educación Artística [9].
La UNESCO (Organización de las Naciones Unidas para la Educación, la Ciencia y la Cultura) ha organizado diversas conferencias mundiales sobre Educación Artística, de las cuales ha quedado como conclusiones que desde hace medio siglo la

Educación Artística sigue sin cambios significativos, aun a sabiendas del desarrollo de la capacidad creativa que esto representa[10], ahora impregnado de dos nuevos matices: uno, considerando creativas no solo a las personas sino también a las instituciones e industrias, y por otro vinculándola a los nuevos contextos sociales:

"Las sociedades del siglo XXI exigen cada vez más trabajadores creativos, flexibles, adaptables e innovadores y los sistemas educativos deben ajustarse a esta nueva situación. La educación artística constituye asimismo un medio para que los países puedan desarrollar los recursos humanos necesarios para explotar su valioso capital cultural. La utilización de estos recursos y este capital es vital para los países si desean desarrollar industrias e iniciativas culturales fuertes, creativas y sostenibles, las cuales pueden desempeñar un papel clave al potenciar el desarrollo socioeconómico en los países menos desarrollados" [11].

Todo ello muestra en qué medida se han ampliado los contenidos y alcances de la educación artística, y la trascendencia de la pregunta realizada inicialmente, en cuanto al lugar que ocupa la educación artística dentro de la educación básica, y el conjunto de materias educativas que la integran, de manera que sean capaces de explicar el mundo actual, lo cual tambien expresa el desafio de nuestros programas y el de la propia practica pedagógica en torno a una formación integral de los escolares.

El modelo escogido en cuanto a favorecer la creatividad y no la condición estética per se de las artes, rasgo común de las concepciones que predominan, responderá por si sola a la meta de convertir la educación artistica en un instrumento pedagógico en el proceso enseñanza-aprendizaje de los escolares.

Sin embargo ya se sabe que dicho paradigma no ha conseguido imponerse de igual manera en la práctica de los modelos pedagógicos. En general ello podría deberse a diferentes factores de orden económico, administrativo y hasta procedimental, pues no siempre resulta fácil introducir cambios en los 
programas establecidos, sobre todo en el ámbito de la educación pública, por lo que de hecho a llegado a ser lentamente gradual el triunfo de esta perspectiva de orden cualitativa, que sustenta la meta mundial de una educación artística de calidad, pero que aún tiene pendiente también el problema del cómo transformarla en un instrumento de desarrollo formativo, vocacional y creativo de la enseñanza de los escolares, lo cual en la opinión del autor ha resultado determinante, en los desempeños irregulares que se han obtenido, dada las dificultades teórico-prácticas, que no han favorecido su instrumentación sostenible, en los sistemas educativos regionales y nacionales.

Las Conferencias mundiales y especialmente las latinoamericanas, en torno a la educación artística, han sido un intento por incidir en esta problemática de la enseñanza. Una de la iniciadoras de ese ciclo de debates, proyectos y gestión programáticas en torno de esta educación, parece estar enlazados a la realizada en Bogotá, del 28 al 30 de noviembre de 2005: "Hacia una Educación de Calidad: Retos y

Oportunidades", realmente una reunión preparatoria de la Cumbre Mundial de la UNESCO sobre Arte y Educación [12].

La Conferencia convocó a 21 países logrando la participación de 18 expertos gubernamentales del sector educativo y de 20 expertos del sector cultural, quienes representaron a 20 Estados de la región. En la fase de preparación, el Comité Académico del evento hizo llegar a los países una guía para la exposición en paneles y mesas redondas correspondientes a tres ejes temáticos de reflexión: La Educación Artística en las Políticas Culturales y Educativas, La Formación de los Educadores y la Configuración de su Saber Artístico y Implicación de los Artistas de la Comunidad y de las Instituciones Culturales en el Proceso Educativo.

Así mismo se envió el Documento Conceptual elaborado por la Oficina Regional de Cultura de la UNESCO y un Cuestionario [13], para ser diligenciado conjuntamente por los delegados de educación y cultura, cuyos resultados permitieron obtener una visión global sobre la Educación

Artística a nivel de la región.

Aquel cuestionario ha sido un pretexto metodológico para realizar una mirada local a esta problemática, pues los pronunciamientos de la llamada
"Declaración de Bogotá", siguen aún vigentes en los marcos de la revolución ciudadana, que pretende la formación de mejores ciudadanos. Además de inspirar y constituir los fundamentos de las Metas Educativas 2021, centro de los debate sobre Educación artística, Cultura y Ciudadanía y del programa que tiene la intención de coordinar en Iberoamérica las propuestas contenidas en las Conferencias Mundiales y regionales convocadas por la UNESCO, en la Conferencia de Lisboa 2006 y su hoja de ruta [14], y especialmente las recomendaciones y lineamientos emanados del Congreso de Formación Artística y Cultural para la Región de América Latina y el Caribe celebrado en Medellín en 2007 [15], elementos que revitalizan aquel instrumento metodológico, que nosotros también adaptamos a los objetivos y entorno concreto de estas ciudades.

Como ha ocurrido en otras partes, entre nosotros la educación artística se asocia a la formación de una cultura estética, y ello no sería una limitante de no haberse manifestado dificultades para enseñarla, en virtud de que la mayoría de los docentes no están preparados metodológicamente. Las materias con "más fuerza" luchan para conseguir más horas para ellas en un apretado horario, y sobre todo al momento de impartirlas solamente se reduce a seguir modelos, lo cual ocasiona que el niño no amplíe su capacidad productora [16].

El predominio de esta dificultad, es el primer indicador de que mucho tiene que revelarnos este cuestionario, en cuanto al estado de insercion de la educación, artística, ello además develara las reales dimensiones que posee el modelo pedagógico de nuestras escuelas, en cuanto a fomentar una formación transformadora del mundo, que debe ser construido por la próximas generaciones de ecuatorianos.

\section{Título de la primera sección.}

Para efectuar el estudio se adaptó la encuesta de la UNESCO, arte y educación aplicada en los países latinoamericanos, cuyos resultados fueron analizados en la Conferencia Regional de América Latina sobre Educación Artística "Hacia una Educación Artística de Calidad: Retos y Oportunidades", en noviembre del 2005 [17], el cuestionario en mención sobre educación artística, se aplicó a una muestra aleatoria de docentes en ejercicio de escuelas de educación básica, pertenecientes al sistema de la educación 
pública, pues es el ámbito donde hemos interactuado con la práctica docente, ya sea como maestro o asumiendo diversas funciones de la actividad pedagógica. A pesar de conocer este universo, resultó complejo elegir una muestra representativa, debido a que los recursos logísticos, así mismo la dinámica docente complicaba la incorporación de un mayor número de potenciales expertos y maestros.

Los que consintieron formar parte del proyecto de investigación, aceptando nuestras condiciones, se integraron por razones de conciencia, al necesitar una retroalimentación del impacto del trabajo que realizan, ya que este tipo de estudio efectuado por la carrera de Artes de la Universidad Técnica de Machala, fue presentado en los debates de preparación metodológica que organiza esta carrera, la cual posee foros de discusión virtual especializado, para la organización y comunicación interna, dado que posee una especial capacidad de convocatoria y confrontación con el gremio de profesionales de la enseñanza de este nivel de educación.

Fue natural elegir como recurso metodológico el foro virtual, en sus diversas modalidades; tales como El Foro Discusión (abierto) y El foro grupal (más cerrado). Ambos permitieron la confrontación de opiniones y experiencias, sobre los diferentes temáticas sometidas a debate por el cuestionario; adaptándolo a las condiciones e intereses de las autoridades rectoras de la enseñanza básica y media en éstas ciudades ecuatorianas, Pasaje y Machala.

\section{El Primero de los foros reunió a maestros con} experiencia que oscila entre los 10 y 20 años, el segundo, para participar en él, debía pertenecer al sistema de gestión educativa de los sectores en estudio, se agruparon a los funcionarios del sistema educacional de las ciudades mencionadas, metodólogos, directores, asesores y mentores, por lo que, la caracterización que presentamos, se realiza desde dentro, o sea, desde la práctica directa de maestros, y desde fuera, o sea extra-docente, al tener en cuenta la mirada organizativa y administrativa, de quienes toman las decisiones y priorizan las políticas de desarrollo, por lo que se trata de un imaginario lo más completo posible, de los agentes involucrados con algún grado de participación, en la misión de garantizar una educación artística de calidad de nuestros estudiantes.
El cuestionario posee una naturaleza abarcadora, de todos los factores que operativa y funcionalmente afectan la eficacia del programa de la educación artística, algunas preguntas se comportan como ejercicios técnicos, capaces de filtrar la real experticia de maestros y funcionarios. Por lo que puede advertirse que no se trataba de un simple sondeo, ni una encuesta, sino un cuestionario a profundidad, para un público experto en el tema, y entrenado para realizarlo.

De modo para su aplicación resultaba determinante, que no se perdiera la motivación y permanencia de los participantes, en contacto virtual a través de la red que habitualmente conecta a estos profesionales, que durante un mes, debían mantener la confrontación de opiniones, debate necesario para obtener un diagnóstico participativo.

De esta manera se confeccionó un cronograma de aplicación, cuyas etapas fueron las siguientes: Publicación de las características, y condiciones de la realización del foro, así como el listado oficial de participantes; anuncios promocionales general e individual sobre el Foro de la Enseñanza Artística; publicación gradual de los cuestionarios; monitoreo diario de los debates y recogida de la información; publicación diaria de los resultados parciales, para estimular la confrontación; publicación de las Conclusiones Preliminares; elaboración y entrega de Informe Final

En general metodología y procedimientos están encaminados a garantizar, la aplicación más conveniente, que garanticen la obtención de resultados, lo más cercano posible a la experiencia de las personas, pues se trataba de un cuestionario armado para tales efectos. Los cambios introducidos, están referidos al lenguaje para hacerlo funcional, exento de tecnicismos que complicaran la impronta de los debates, y la agilidad para procesar los datos.

\section{Resultados y discusión}

El análisis que presentamos es solo una aproximación al estado de la educación artística, en Pasaje y Machala. El estudio logró convocar a 76 profesionales de educación básica y artística, quienes representan el 17,6\%, de 432 docentes, de 36 escuelas seleccionadas para el estudio, de un total de 243, dato extraído de AMIE, [18].

\section{Procedimiento}


Este instrumento contenía 23 preguntas estructuradas en cuatro ejes: 1) Caracterización Básica; 2) Perfil de maestros y estudiantes; 3 )

Políticas-objetivos; 4) Financiación

Para el análisis de los resultados se asumirá el 100\% como el total de los 76 maestros que participaron en el estudio.

\subsection{Caracterización Básica}

La educación artística es obligatoria en educación básica y en secundaria, según acuerdo Ministerial, 00064-A, [19], que regula 2 horas semanales para octavo, noveno y décimo de Educación Básica, así como para primero y segundo de bachillerato. Los encuestados como promedio reportaron que el número de horas semanales impartidas en educación básica es de 2 horas; ello, es coincidente con las conclusiones del informe general de la UNESCO, el cual reporta que ningún país de los encuestados dedica 6 horas a la educación artística.

Las áreas artísticas por nivel y por modalidad incluidas en la educación, se comportó como sigue. El $84 \%$, afirman que la música, la pintura y el dibujo constituyen las áreas habituales del currículo; 16\% refiere que incluyen danza, teatro y literatura. Interesante indicador, pues en los países de la región una mayor proporción de países incluyen el área de literatura $82 \%$.

En lo que hace referencia a las áreas artísticas que dan mayor énfasis en la cultura tradicional. El $14,2 \%$ de los encuestados consideran que es la Danza, el 14\%, las Artesanías, y el 13,7\% la Música y el $10,2 \%$ la Literatura, cuestión que resulta interesante, pues son los elementos más fuertes de la tradición cultural ecuatoriana, que sin embargo no ha sido regulado por ningún decreto, pero si en consonancia con la política cultural, de conservar los elementos autóctonos de cada comunidad.

-Se consideró también importante, tener una idea aproximada, de los objetivos que tienen mayor énfasis en la educación artística. El 33,2\% de las muestras encuestadas indica que la educación artística tiene mayor énfasis en los Objetivos Culturales, seguido por Objetivos Sociales $23,1 \%$, Objetivos Artísticos/Estéticos 22,5\%, Vocacionales $9,5 \%$, Económicos 6,7\%, Alfabetización 3,1\% y el $1,8 \%$ señala que son Otros los Objetivos.

\subsection{Perfil de maestros y estudiantes}

Necesitábamos definir el nivel de compromiso de quienes, tenían a su cargo la responsabilidad de impartir o gestionar este programa, y conocer cómo evaluaban la educación artística ya sea cómo componente o complemento de la enseñanza básica.

El $95,49 \%$ de los participantes consideraron que la educación artística es un componente fundamental, el $4,5 \%$ la consideró un complemento de la enseñanza básica, lo cual quiere decir, que se ha avanzado en cuanto a la importancia concedida a la educación artística en la enseñanza general, pero, es significativo el hecho que entre profesionales del ramo exista un índice que se resista de alguna manera, a considerar su importancia de manera consciente. Por lo que ello apunta que debe trabajarse más en cuanto a la formación de los docentes en tal sentido.

Así mismo, ante la pregunta del papel del arte en el desarrollo de la creatividad del niño. La respuesta fue casi unánime. El 92,7\% de los encuestados manifestó estar de acuerdo y 7,3\% señaló desacuerdo. Lo que implica que ambas preguntas solo hacen ratificar, lo ya dicho en torno a la formación de los maestros.

Fue interesante cruzar esas respuestas con el perfil que conformaban los maestros que participaron en el cuestionario. El $46 \%$ fueron docentes que imparten educación artística en los establecimientos públicos, $35 \%$ son funcionarios y $19 \%$ son generalistas complementan la tarea de los docentes. Sin embargo los perfiles tienden a ser variados, como regla son los docentes los que llevan a cabo el programa, lo cual los convierte en centro y parte de las problemáticas con la enseñanza.

\subsection{Política y objetivos}

Nos interesaba que la encuesta expresara de alguna manera el impacto y problemáticas de políticas en materia de educación artística. Esta pregunta y otras que continúan revelan, de hecho potenciales objetivos estratégicos en el futuro. ¿Quiénes de estas instancias intervienen en los contenidos impartidos en el programa de la enseñanza artística?

El $100 \%$ de los encuestados expresaron que el gobierno central es el principal encargado de definir contenidos para la educación básica, así como la 
educación en general, ello indica que los gobiernos locales no tienen incidencia, así como las familias y estudiantes.

- El $70,5 \%$ de los encuestados piensan que las artes son parte integral de una formación educacional completa, el $19,4 \%$ señala que quizás, mientras que el $10,1 \%$ indica lo contrario.

- ¿Cuáles de las siguientes áreas artísticas considera usted que deberían estar incluidas en la educación artística a nivel básico?

De los resultados se obtuvo que la mayoría de los encuestados considere que la Pintura $66 \%$, al igual que el Dibujo 12,2\%, la Música $11,5 \%$ y la Literatura $10,3 \%$ deberían estar incluidas en la educación artística a nivel básico.

¿Existe espacio en los medios masivos de comunicación especialmente concebidos para apoyar programas de educación artística?

El 37,6\% de las muestras encuestadas señala que Sí existe un espacio en los medios masivos de comunicación especialmente concebidos para apoyar programas de educación artística, mientras que el $62,4 \%$ señala lo contrario.

¿Cuál o cuáles son los medios de comunicación concebidos en apoyar los programas de educación artística?

El $50 \%$ de los encuestados indica que el mayor medio de comunicación concebido en apoyar los programas de educación artística son los Programas de Televisión, mientras que los Programas de Radio, Separatas en los periódicos y Otros abarcan el otro $50 \%$ conjuntamente.

¿Sabe usted que la educación ejerce un impacto mensurable en la reducción de las conductas delictivas y la inasistencia escolar de los jóvenes con alto índice de riesgo?

El $80,6 \%$ de las muestras encuestadas indica que si poseía conocimientos de que la educación ejerce un impacto mesurable en la reducción de las conductas delictivas y la inasistencia escolar de los jóvenes con alto índice de riesgo, mientras que el 19,4\% señala lo contrario.
- ¿Sabe usted que las habilidades artísticas se relacionan directamente con índices reducidos de deserción escolar, especialmente en niños con desventajas económicas?

El $77,9 \%$ de los encuestados indica que si poseía conocimientos de que las habilidades artísticas se relacionan directamente con índices reducidos de deserción escolar, mientras que el $22,1 \%$ señala lo contrario.

- ¿Existe un programa gubernamental de publicación de documentos (investigaciones, guías metodológicas, etc.) dedicados a la educación artística?

El 55\% de las muestras encuestadas desconoce que exista un programa gubernamental de publicación de documentos dedicados a la educación artística, mientras que el $45 \%$ se encuentra seguro de que sí existe.

- ¿En qué medida valora usted a las áreas artísticas en la educación?

El $37,8 \%$ de las muestras encuestadas valora en el nivel alto a las áreas artísticas en la educación, el $45,8 \%$ las ubica en el valor medio, mientras que el $16,3 \%$ las consideran en el nivel bajo. Por lo que se infiere la necesidad de programas especiales que incidan en mejorar la práctica de la enseñanza.

\subsection{Financiación}

- Esta pregunta fue considerada clave pues era un filtro para determinar el nivel de compromiso de las autoridades en el fomento de la educación artística. Resulta llamativo que los encuestados no refirieron conocimiento alguno respecto a presupuestos específicos, aunque si consideraron la financiación general de la educación que realizan los gobiernos centrales, provinciales y locales.

\section{Conclusiones}

Lo analizado muestra en qué medida se han ampliado los contenidos y alcances de la educación artística, y la trascendencia de las preguntas realizadas inicialmente, en cuanto al lugar que ocupa la educación artística dentro de la educación básica, y el conjunto de materias educativas que la integran, de manera que sean capaces de explicar el mundo 
actual, lo cual tambien expresa el desafio de nuestros programas y el de la propia practica pedagógica en torno a una formación integral de los escolares.

La aproximación iniciada en el entorno de estas ciudades ecuatorianas, apunta que las localidades son un reflejo de la necesaria profundidad de las políticas socio-culturales, y en ese entorno hay mucho que hacer en materia educativa. Sin embargo la excesiva centralización de aspectos de la cultura que se vuelven "mediáticos", puede impedir la real participación de estos espacios, pues poco se deja a la introducción de la autoctonía local, y ello, es una problemática compleja en la culturalidad del Ecuador.

Otro aspecto importante que arroja el presente estudio es referente a las prácticas docentes, necesitadas de mejores formadores, mayores debates donde se sistematicen nuestras buenas prácticas, además de promover los estudios que indaguen sobre cómo, convertir la educación artística, desde la plataforma inclusiva que promueve el "buen vivir", y el creciente desafío de edificar el hombre nuevo, que sepa cultivar su identidad propia, en el contexto de un universo cada vez más exigente en cuanto a conocimiento e interacción multicultural.

Por otro lado, la valoración efectuada permitió mostrar que la estructura curricular de educación básica ecuatoriana, desde primer año hasta séptimo no contempla la enseñanza de las artes, situación preocupante sobre todo si se considera que las respuestas obtenidas en la pregunta que hace referencia, "si las artes son parte integral de una formación educacional completa", cuyo resultado fue unánime de que es primordial complementar la educación general con la educación artística, lo que sin lugar a duda se constituye en imprescindible incorporar la enseñanza de las artes en edades tempranas para alcanzar una formación integral en el niño a través de una educación de calidad, para todos los ciudadanos de la patria.

Aunque los resultados obtenidos de los consultados sobre el número de horas a la semana dedicadas a las actividades artísticas, quienes coinciden en que son dos horas, quizá la referencia se deba a que en Octavo, Noveno y Décimo de Educación Básica, si reciben esas dos horas. Lo que deja entrever que los demás grados no cuenta con la enseñanza artística, dejando de lado actividades indispensables en el desarrollo integral del niño, sobre todo en edades tempranas, situación que debe llevar a realizar análisis profundos en los diferentes estamentos educativos con el fin de lograr comprender la realidad y así poder dar respuesta a esta necesidad educativa.

\section{Agradecimientos}

Expreso mi profundo agradecimiento a los estudiantes de primer año de la Carrera de Artes Plásticas de la Universidad Técnica de Machala, por su excelente labor en el desarrollo del Foro. Considero que sin su entusiasmo y apoyo no hubiese sido posible el mismo; de la misma manera al Mgs. Erwin Peñaherrera, coordinador de la carrera, por la apertura que dio al evento como a la participación en el mismo; además a los maestros y directivos de las 36 escuelas que participaron de manera activa en todo momento con sus ideas para dar respuesta a las interrogantes propuestas.

\section{Referencias}

[1] M. Acaso, La Educacion Artistica No Son Manualidades: Nuevas Prácticas En La Enseñanza de las Artes y La Cultura Visual, Editorial La Catarata, (2014), p. 25

[2] UNESCO, Hoja de Ruta para la Educación Artística, Conferencia Mundial sobre la Educación Artística: construir capacidades creativas para el siglo XXI, Lisboa, 6-9 de marzo de (2006), pág.2

[3] UNESCO, Hoja de Ruta parala Educación Artística, Conferencia Mundial sobre la Educación Artística: construir capacidades creativas para el siglo XXI, Lisboa, 6-9 de marzo de (2006), pág.3

[4] V. Lowenfeld, La Naturaleza de la Actividad Creativa, (1939), p.37.

[5] M. Barkan, et al, Desarrollo Curricular para la Educación Estética, San Luis. Missouri: Laboratorio Educativo central del medio oeste Regional, (1970), pp. 56-61

[6] R. Marín, Las investigaciones en educación artística y las metodologías artísticas de investigación en educación: temas, tendencias y miradas, Porto 
Alegre, vol. 34, No. 3, (2011) pp. 271-285.

[7] M. Hernández, El arte de los niños. Investigación didáctica del MUPAI. Madrid: Fundamentos, (1995), p. 121

[8] L. M. Martínez G, Arte y símbolo en la infancia. Barcelona: Octaedro. (2004), p. 52

[9] R. Marín, Las investigaciones en educación artística y las metodologías artísticas de investigación en educación: temas, tendencias y miradas, Porto

Alegre, vol. 34, No. 3, (2011) pp. 271-285.

[10] V. Lowenfeld, La Naturaleza de la Actividad Creativa, (1939), pp. 23-25

[11] UNESCO, Hoja de Ruta para la Educación Artística, Conferencia Mundial sobre la Educación Artística: construir capacidades creativas para el siglo XXI, Lisboa, (2006), pág. 4.

[12] CONFERENCIA REGIONAL DE AMÉRICA LATINA Y EL CARIBE SOBRE EDUCACIÓN ARTÍSTICA, "Hacia una educación artística de calidad: retos y oportunidades"

portal.unesco.org/culture/es/files/31272/.../rui z_correal_colombia.doc (2005), P. 1-4

[13] https://www.lacult.org/docc/CUESTIONAR IO_UNESCO.doc

[14] UNESCO, Hoja de Ruta para la Educación Artística, Conferencia Mundial sobre la Educación Artística: construir capacidades creativas para el siglo XXI, Lisboa, (2006), pp. 3-4.
[15] La Educación que Queremos para la Generación de los Bicentenarios, Metas Educativas 2021 , http://www.oei.es/metas2021/foroart.htm, Medellín (2007), p. 1-2

[16] V. Lowenfeld, El niño y su Arte, Editorial Kapelusz, Buenos Aires, (1946) p. 12.

[17] Conferencia Regional de América Latina y el Caribe Sobre Educación Artística, "Hacia una educación artística de calidad: retos y oportunidades", portal.unesco.org/culture/es/files/31272/.../rui z_correal_colombia.doc, (2005), P. 1-4

[18] AMIE, Archivo Maestro de las Instituciones Educativas, Ministerio de Educación del Ecuador, http://reportes.educacion.gob.ec:8085/inde x.aspx, 2015. P. s.p.

[19] Ministerio de Educación del Ecuador, ACUERDO Nro. MINEDUC-ME-201400064-A, http://educacion.gob.ec/wpcontent/uploads /downloads/2014/12/MINE DUC-ME-201400064-A.pdf. (2014), p. 4.

E-mail: Jubonista15@hotmail.com 